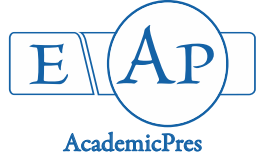

\title{
The Role of DNA Methylation in Perennial Plants
}

\author{
Lia BASDEKI, Marianna HAGIDIMITRIOU* \\ Agricultural University of Athens, School of Food, Biotechnology and Development, Department of Biotechnology, \\ GeneticsLaboratory, 75 Iera Odos 11855, Athens, Greece; labsdeki@gmail.com; marianna@aua.gr ('correspondingauthor)
}

\begin{abstract}
DNA methylation is an important epigenetic modification of the genome in all organisms. This review presents the effect of DNA methylation in perennial fruit trees such as chestnut, apple, peach etc. In particular, DNA methylation has been shown to affect bud dormancy, the changes of developmental stages such as: flowering, the synthesis of anthocyanins, among other flavonoid compounds; it also affects the fertilization and perpetuation of many fruit trees. Finally, DNA methylation can be used as a tool for investigating the epigenetic diversity of a species.
\end{abstract}

Keywords: anthocyanins; dormancy; fruit trees; 5-methyl-cytosine

\section{Introduction}

The term "epigenetics" was used and described for the first time in 1942 by Conrad Waddington. Originally it was used for describing the occasional interactions between genes and their products, which are capable of leading to phenotypic expression. Nowadays, "epigenetics" describes all inherited phenotypic changes due to processes that take place independently of the original DNA sequence (Chong et al., 2004; Liu et al., 2008; Jin et al., 2011). The epigenetic process can be transferred to organisms from one generation to the next (Tollefsbol, 2011). Epigenetic regulation of gene expression is achieved by methylation of DNA, histone or chromatin modifications, chromatin remodeling, etc. (Reinders et al., 2015).

The term methylation refers to the covalent transfer of a methyl group $\left(\mathrm{CH}_{3}\right)$ to the $5{ }^{\circ} \mathrm{C}$ of the cytosine ring of DNA (Jin et al., 2011). This addition takes place during the formation of each polynucleotide chain of DNA by specific enzymes, DNA methyltransferases (DNMTs) (Molfetas et

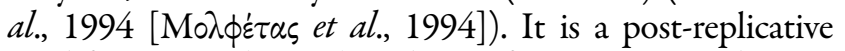
modification as the nucleotides are first incorporated into a DNA strand and then altered by the addition of the methyl group.

Methylation is reported in many organisms including prokaryotes, fungi, plants and animals. Although it is found in all of these organisms, a different function is believed to be exhibited for every single one of them (Doerfler and Bohm, 2006). Methylation involves a significant chemical modification of DNA, which does not result in a change in the genetic code sequence, but has the potential to be inherited through mitotic and meiotic divisions in plant genomes (Niederhuth and Schmitz, 2013) and is characterized as an epigenetic phenomenon.

In the 1960s, two possible actions were proposed for DNA methylation. According to the first hypothesis, methylation is involved in a protection mechanism against foreign DNA integration. The second hypothesis attributes resistant DNase to the DNA of the host which is directed against foreign DNA (Srinivasan and Borek, 1964). It is now known that DNA methylation is involved in many cellular processes such as transcriptional and pericentromeric domain silencing, $\mathrm{X}$ chromosome inactivation and genomic imprinting. It is a relatively stable epigenetic signal, which is still regulated dynamically; this results from the fact that in order to maintain methylation at specific levels, it is necessary to correctly maintain the methyltransferases of the DNA during replication.

From an evolutionary perspective, methylation of DNA is believed to represent an ancient mechanism and in most eukaryotes it consists of the transfer of a methyl group from SAM (S-adenosylmethione) to the fifth end of the cytosine to specific $\mathrm{CpG}$ dinucleotides. This process is catalyzed by a group of enzymes called DNA methyltransferases (DNMTs). Their catalytic domain is conserved from prokaryotes to humans (Kumar et al., 1994). A general rule that has been observed states that, the more methylated the gene regulation region is the more likely it is that the gene activity is reduced and vice versa. Of course, there are some exceptions to this rule (Tollefsbol, 2011). 
At genome level, methylation is remarkably stable. In particular, the methylation of transposable elements is the most stable and consistent of all, as demonstrated by an experiment in 30 consecutive plant generations in Arabidopsis thaliana (Niederhuth and Schmitz, 2013). Experiments have shown high levels of methylation at CpG sites of angiosperms. Comparisons between slowly evolving species showed particularly high levels of maintenance of methylation between their orthologue genes. In addition, these comparisons suggest that methylation and silencing of transposable elements is an ancient process for plants (Niederhuth and Schmitz, 2013). This feature is confirmed by Barghini et al. (2014), who studied a type of retrotransposable element in the olive genome. Their research has focused on SINEs (Short Interspersed Nuclear Elements), which are retrotransposable elements of eukaryotic organisms and are believed to precede the appearance of LTRs (Long Terminal Repeats). According to a theory, these two types of retrotransposable elements appeared and reinforced their presence in the genome since ancient times, and after their replication and translocation the methylation mechanism appeared.

In the genomes of many eukaryotes lies a large proportion of repetitive DNA sequences, which can either be organized in tandem arrays or in dispersed fashion. The length of tandem DNA repeats may vary from two to many thousands of base pairs (Flavell, 1986), while a large number of dispersed repeats consists of mobile genetic elements (Smyth, 1991). Katsiotis et al. (1998) attempted to describe the genomic and chromosomal organization of $O$. europaea ssp. sativa; for that purpose a partial Sau3Al library was constructed. From this library, a highly repeated tandem DNA family - the 81bp Sau3Al family - as well as another tandemly repeated DNA clone - pOS218 - were isolated and studied by Southern and in situ hybridization. Their results indicate that the tandem arrays of both elements are largely methylated at their cytosine residues (Katsiotis et al., 1998). Furthermore, Stergiou et al. (2002) showed that Olea retrotransposon sequences are also methylated.

Methylation is related to gene transcription as well as to chromatin structure. In the case of plants, DNA methylation is closely related to histone modifications. It also appears to affect the binding of specific proteins to DNA as well as the formation of the corresponding transcription complexes in chromatin. According to Bitonti et al. (2002), methylation is believed to be responsible not only for regulating the level of gene expression but also for maintaining epigenetic changes. These features have led to the understanding that methylated cytosine is a very good candidate for the control of genes whose expression is essential for the development of a particular cell type.

According to Hasbún et al. (2007), DNA methylation can act as a molecular marker for specific plant growth processes. These processes may be related to aging, regeneration as well as to the changing growth stages of plant development. These functions concern both the gymnosperm and the angiosperm plants. Aging was associated with increased content of methylated cytosines. The high rate of methylation during bud dormancy was followed by a decrease in bud differentiation. The research team, analyzing these data, was led to the conclusion that bud dormancy is accompanied by a general increase in methylation regardless of the plant's age. They also confirmed that methylation is involved in DNA transcription, as it can lead to gene silencing. During dormancy, meristems remain inactive due to such modification in DNA transcriptional activity. It seems that methylation directly affects the normal growth of plants, which is supported by the abnormal growth of plants in the event of changes in their methylation pattern (Hasbún et al., 2007).

Bitonti et al. (2002) moving in similar context with Hasbún et al. (2007) examined changes in the levels of methylation in meristems of peach trees. The meristems were divided into three groups: juvenile, juvenile-like and adult. It was observed that during the vegetation development of peach (March-June), the levels of methylation of nuclear DNA were higher in adult meristems than in juvenile and juvenile-like. Thus, some key effects of methylation, such as its involvement in gene regulation and expression as well as the changes it brings to the structure of chromatin, have been confirmed. Changes in methylation levels were also observed during the changing of developmental stages in peach. Bitonti et al. (2002) concluded that changes in DNA methylation levels either regulate the change of the growth phases in higher plants or simply occur during these periods.

These conclusions are reinforced by the action of genomic imprinting and the recognition of epigenetic activity in organisms. The genomic imprinting also has an epigenetic base and occurs in plants by analogous processes to the mammalian ones. It may occur in certain types of tissue or during developmental stages that play a critical role in the development of an organism. It is not by chance that epigenetics plays such an important role in the evolutionary creation of new species as well as in the effect of mutations (Tollefsbol et al., 2011).

Methylation of cytosines in eukaryotic genomes has often been associated with repeated sequences, including transposable elements and their derivatives. Such sequences are often found in centromeric and pericentromeric regions (Reinders et al., 2015). These repeated sequences could have two possible harmful effects which are controlled by methylation. Methylation often prevents uncontrolled transcription of genes which may disrupt the expression of other neighboring genes. It also controls potential changes in the genome structure by translocation and ectopic recombination. The loss of methylation is considered sufficient for the mobilization of transposable elements and their possible negative effects (Kato et al., 2003; Tahilliani et al., 2009).

\section{CpG islands, $\mathrm{CpHpG} \& \mathrm{CpHpH}$ sites}

Methylation of cytosine residues occurs predominantly in $\mathrm{CpG}$ dinucleotides and in specific regions of the genome called $\mathrm{CpG}$ islands. The CpG sequences contain a higher percentage of cytosines and guanines than average. CpG dinucleotide islands (cytosine - C - joined by $3^{3}-5^{\prime}$ phosphodiester bond to guanine - G) are about $1-2 \mathrm{~kb}$ in length, are often near transcriptional start points and when methylated they suppress the expression of their closest 
genes (Nikolaou, 2015).

Unlike mammals, methylation in fungi and plants is not limited to $\mathrm{CpG}$ sites; $\mathrm{CpHpG}$ sequences may also be methylated (Doerfler and Bohm, 2006). Although previous studies have shown that there is a correlation between $\mathrm{CpG}$ islands and methylated cytosines, it is now known that in plants, DNA methylation is frequently found in $\mathrm{CpG}$, $\mathrm{CpHpG}$ and $\mathrm{CpHpH}$ residues, with $\mathrm{H}$ representing one of the following: A (adenine), $\mathrm{T}$ (thymine) or $\mathrm{C}$ (cytosine) (Kavarik et al., 1997; Lister and Ecker, 2009; Niederhuth and Schmitz, 2013).For instance, in Arabidopsis thaliana methylation was detected in all $\mathrm{CpG}, \mathrm{CpHpG}$ and $\mathrm{CpHpH}$ sequences. The highest percentage of methylation was observed in CpG-type residues (24\%), followed by $\mathrm{CpHpG}$ sites (6.7\%), and $\mathrm{CpHpH}$ sites (1.7\%). The differences observed in the methylation of these different groups of nucleic acid residues underline the different genetic control exercised in each of these cases (Cokus et al., 2008).

Small interfering RNAs -siRNAs- are groups of double stranded RNA molecules, 20-25bp long, that are involved in many biological pathways such as the RNAi (RNA interference) pathway. In plants, siRNAs can lead to DNA methylation of some genetic loci of high homology to their sequence. The siRNAs arise from the same sequences that will methylate later on via RdDM pathway (RNA directed DNA Methylation) (Xie and Yu, 2015). The RdDM pathway is mainly involved in asymmetric methylation points $(\mathrm{CpHpH})$ and in very rare cases symmetric methylation sites (CpG). Typically, $\mathrm{CpG}$ and $\mathrm{CpHpG}$ sites are methylated through other mechanisms that involve the enzymes MET1 (Methyltransferase 1) and CMT3 (Chromomethylase 3) (Xie and $\mathrm{Yu}, 2015$ ).

The methylation of $\mathrm{CpG}, \mathrm{CpHpG}$ and $\mathrm{CpHpH}$ sites is mainly located in pericentromeric regions, which contain a high percentage of repetitive sequences. CpG methylation is almost exclusively found in the main part of the genes and its percentage is higher than that of $\mathrm{CpHpG}$ and $\mathrm{CpHpH}$ methylation (Cokus et al., 2008). The fact that methylated CpG sites are found in the main part of the genes is in agreement with the limited presence of siRNAs in those regions (Cokus et al., 2008).

\section{Methylation in plants}

Approximately 1-6\% of the nucleotides in plant and animal genomes consist of 5-methyl-cytosine, commonly known as DNA methylation. Plants exhibit several methylation differences in comparison to animals. To begin with, cytosine methylation in plants is richer than that of the animals' genome. In addition, methylation in plants is encountered in three different groups of base residues, in contrast to animals in which methylation occurs exclusively in CpG islands (Cokus et al., 2008; Lister and Ecker, 2009).

DNA methylation is established de novo in plants with the orthologue genes Domains Rearranged Methyltransferase $1 / 2$ also known as DRM 1/2. The following methyltransferases are also found in plants: MET1, DRM1, DRM2 and CMT3. These genes target specific methylated loci with small RNA molecules in the context of RNA directed DNA methylation. MET1 is equivalent to
DNMT1, which is found in animals, and is responsible for the post-replicative maintenance of DNA methylation in CpG sites of plants. Loss of MET1 leads -amongst other things- to the near-complete deletion of $\mathrm{CpG}$ methylation, the suppression of demethylation processes, the change of RNA directed methylation activity and the redistribution of other suppressive epigenetic mechanisms (Reinders et al., 2015). Chromomethylase 3 (CMT3) is essential for the maintenance of DNA methylation in $\mathrm{CpHpG}$ sites in plants.

Methylation of $\mathrm{CpHpH}$ sites is abundant in plants and is maintained by the RdDM pathway that targets the DRM2 (Domains Rearranged Methyl-transferase 2), which is homologous to DNMT3 found in animals (Cokus et al., 2008; Jin et al., 2011; Niederhuth and Schmitz, 2013). $\mathrm{RdDM}$ is responsible for maintaining the methylation of recent DNA replicated sites. In plants, it prevents the entire genome being uncontrollably and simultaneously replicated. It also causes gene silencing until co-expression with other genes is necessary or their re-expression or their ultimate absorption into the genome (Niederhuth and Schmitz, 2013; Zhang et al., 2014). In addition, RNA directed methylation can restore methylation levels after derangement of the organism or its transition from one developmental stage to another (Reinders et al., 2015).

Moreover, plants have demethylation mechanisms. The enzymes ROS1 (Repressor or Silencing 1), DME (Demeter), DML2 (Demeter Like 2), and DML3 (Demeter Like 3) recognize and remove methylated cytosines resulting in nucleotide blanks on the double stranded DNA. Afterwards, DNA repair and integration enzymes theoretically fill the voids with unmethylated cytosine nucleotides (Cokus et al., 2008; Lister and Ecker, 2009; Jin et al., 2011; Niederhuth and Schmitz, 2013). ROS1, DML2 and DML3 enzymes function mainly in stem tissues and genomic studies have shown that they demethylate hundreds of targeted areas along the genome with a preference in gene regions (Penterman et al., 2007; Lister and Ecker, 2009). The DME protein is mainly expressed in the central cell, its expression decreasing after fertilization and is not detected in the male reproductive organ stamen, or the male gametophyte, pollen; thus only the maternal genome is demethylated leading to specific expression of genes derived from the maternal allele in the endosperm (Huh et al., 2008). This specific activation of genes in female germ cells due to the action of the DME protein, is called imprinting.

According to Lister and Ecker (2009), a periodicity of 167 nucleotides between the methylation points has been detected. This distance (bp) is very close to the distance (bp) between the nucleosome binding sites in plants. This fact indicates that the nucleosome-binding sequences are more exposed to DNA methyltransferases or that methylation is functionally related to nucleosome placement. Recent studies have shown that nucleosomal DNA is an important substrate for DNMTs in vivo (Jin et al., 2011). The same research group sequenced the methylome of a mutant that lacked the enzymes ROS1, DML2 and DML3. Hundreds of distinct hyper-methylated regions were found throughout the genome of that mutant in comparison to the wild-type's genome. The hyper-methylated regions were 
mainly located in primers' and 3' UTRs' regions. These data indicate that demethylases actively protect these domains from methylation, most likely by avoiding the interference of DNA binding proteins or the undesired processes of transcriptional initiation or termination.

\section{Perennial plants}

Perennial trees usually produce fruits that are edible for both humans and animals. In horticulture, the term fruit tree is referred to trees that provide humans with edible fruits, which are known to be rich in vitamins (especially Vitamin A and Vitamin C), minerals, phytochemicals (such as antioxidants) and of course fibers; components essential for a healthy and balanced diet (Slavin and Lloyd, 2012). Pomology, the scientific study of the fruit trees cultivation, divides fruits into groups based on plant morphology and anatomy. Some of those groups are: pome fruits, including apples and pears, and stoned fruits, including peaches, almonds, apricots, cherries and plums (Singha and Baugher, 2003). The development of plants is characterized by juvenile and adult phases. After a relatively short juvenile phase, annual plants progress to the adult phase, during which flowering occurs; perennial plants have a much longer juvenile phase, in some cases it lasts for decades, posing a major obstacle to the genetic improvement (Zhang et al., 2014).

Perennial plants/trees have the ability to suspend and resume growth recurrently in response to environmental or seasonal conditions, a trait of crucial significance to plant productivity and survival. The term dormancy refers to the absence of visible growth in any plant structure containing a meristem and it affects plant productivity, adaptability and distribution. Perennial trees in temperate regions react to the low temperatures and short photoperiod of winter with a period of dormancy. However, the acquisition of sufficient chilling during winter dormancy ensures the normal phenological traits (Kumar et al., 2016; Rothkegel et al., 2017).

Trees use environmental cues to time growth- dormancy transitions in order to balance maximal growth and protect their meristems from frost damage (Santamaria et al., 2011). Bud dormancy is divided into three phases known as paradormancy, endodormancy and ecodormancy (Kumar $e t$ al., 2016). The most critical step is endodormancy, where growth is inhibited by the dormant structure (i.e., the bud). These structures must be exposed to cold temperatures (higher than $4^{\circ} \mathrm{C}$ and lower than $7^{\circ} \mathrm{C}$ ) for a certain number of hours in order to trigger the release of this state (Rothkegel et al., 2017).

DNA methylation is one of the most abundant epigenetic modifications in higher plants and plays a crucial role in regulating developmental processes such as the timing of flowering. The induction of flowering by DNA demethylation has been reported for the low-temperature requiring ecotypes mutants of Arabidopsis (Zhang et al., 2014). Moreover, a reduction of DNA methylation was reported by Hasbún et al. (2007) during the dedifferentiation and cellular reprogramming that takes place in chestnuts' flowering.

Another important trait of perennial plants is their fruit color. The color of the fruit's peel is an important economical trait because it plays a very important role in its market acceptance and value. As far as apples are concerned, red cultivars are preferred and within a particular cultivar the ones that are highly colored are favored despite the fact that consumers' preferences differ from region to region (Telias et al., 2011). However, peel pigments do not only affect the visual appeal but they also contribute to the nutritional value of the fruit due to their antioxidants and bioactive properties (El-Sharkawy et al., 2015). Anthocyanins are a group of water-soluble pigments that confer the blue, purple and red color to many fruits and vegetables. The health benefits of regular intake have been investigated widely and there has been an association between anthocyanins and reduced incidences of cardiovascular disease, diabetes mellitus and cancer (Fang, 2015).

Finally, another important trait is the mechanisms of desiccation tolerance of orthodox (desiccation tolerant) seeds. These mechanisms include the accumulation of late embryogenesis abundant proteins (LEA), dehydrins, nonreducing sugars, sucrose, selenium, and activation of antioxidants, intracellular de-differentiation, metabolic 'switching off, and the operation of repair systems. Orthodox seeds are dried to specific moisture levels before they are stored in gene banks, established to preserve the seeds under conditions that ensure genetic stability and longevity (Michalak et al., 2013).

\section{Bud dormancy}

Bud burst of many trees - including chestnuts- is often dependent on exposure to a particular duration of cool temperatures (chilling) to overcome dormancy, followed by an optimal temperature to permit growth in the spring. An important group of genes involved in entering dormancy are the DAM (DORMANCY ASSOCIATED MADS-BOX) genes which are differentially regulated coordinately with endo-dormancy in buds of several perennial plants. Bud dormancy is fundamental to the study of plant developmental processes and its regulation is of significant economic importance to fruit and horticultural industries (Santamaria et al., 2011). In particular, analysis of methylated cytosine in dormant buds showed a high level of methylation (35\%) (Hasbún et al., 2005). Opposite patterns of acetylated $\mathrm{H} 4$ histone and genomic DNA methylation in dormant and non-dormant buds of chestnut trees have been observed. These patterns provide evidence for different forms of epigenetic control during transition between the phases of dormancy that are likely to control related genes' expression (Santamaria et al., 2011).

During in vitro tissue culture, tissues with juvenile competence undergo a hypomethylation process while mature tissues experiencing a hypermethylation process. Bud dormancy demonstrates a higher methylation level in comparison to active growth period. These data confirmed Hasbún et al. (2005) results since during dormancy the meristems remain inactive due to an alteration in the transcriptional activity of DNA, partially governed by gene silencing which is induced by the methylation of cytosine residues. 
Winter dormancy is a mechanism adopted by temperate plants in order to cope with the low temperatures and the short photoperiod during winter (Kumar et al., 2016; Rothkegel et al., 2017). Apple, being an important temperate fruit crop, requires sufficient chilling so as to overcome winter dormancy which is often associated with the yield and the quality of fruits (Kumar et al., 2016). In chestnut, Hasbún et al. (2005) reported a higher methylation level during bud dormancy compared to active growth period. In apple, Kumar et al. (2016) reported a decrease of total methylation from $27.2 \%$ in dormant buds to $21 \%$ in fruit set state under high chilling conditions; thus demethylation decreased as methylation increased from dormant bud to fruit set stage under low chilling conditions.

In particular, Kumar et al. (2016) performed RNA-seq analysis for 47 genes, which showed correlation between methylation and their expression. These genes are involved in different biological processes and molecular functions. Moreover, bisulfite sequencing and GRT-PCR analysis of selected genes also showed correlation between gene body methylation and gene expression. The results of bisulfite sequencing suggested that low chilling might be associated with hyper-methylation of gene body that might result in suppressing their expression. The total methylation percentage was found to be $24.5 \%$ and $25 \%$ under low and high chill conditions, respectively. The percentage of fully methylated fragments was $19.6 \%$ under high chill conditions and $17.3 \%$ under low chill conditions. The percentage of hemi-methylated fragments was $7.2 \%$ under low chill conditions and 5.5\% under high chill conditions. The chi-square test of independence between chilling availability during dormancy period and methylation patterns suggested association between chilling and methylation level (Kumar et al., 2016).

Another feature investigated by Kumar et al. (2016), was the methylation pattern between the three contexts in which methylation may occur in plants. The three types of cytosine sequence contexts are $\mathrm{CpG}, \mathrm{CpHpH}$ and $\mathrm{CpHpG}$ (where $\mathrm{H}$ represents the $\mathrm{A}, \mathrm{T}$ or $\mathrm{C}$ ). The bisulfite sequenced loci revealed that the maximum number of methylated cytosines was found to be 15 for the CpG type, followed by 13 for the $\mathrm{CpHpH}$ type and 5 for the $\mathrm{CpHpG}$.

\section{Developmental changes (aging, reinvigoration and flowering)}

During their life cycle, trees undergo physiological, biochemical and morphological changes mainly controlled by the alteration of their gene expression; a process that is associated with the change of the morphogenetic capacity and dynamics of epigenetic factors (with DNA methylation being the most known process) (Hasbún et al., 2007). Changes in gene expression caused by alteration of genomic DNA methylation have been related to the loss of morphogenic ability during tree ageing (Hasbún et al., 2005). Juvenile-like and mature primary explants were established in vitro and then analyzed for their methylated cytosines after one proliferation cycle which revealed a similar level of methylation (15\%).

In a multipurpose species, such as Castanea sativa, both juvenile and mature phases may occur at the same adult tree.
The upper parts of a tree are chronologically younger and they often exhibit mature characteristics, whereas the lower parts are physiologically older and they may retain juvenile attributes. The aging of chestnut trees is accompanied with an increase in methylated cytosine residues. Hasbún et al. (2007) associated the shoot apex of juvenile individuals (with no reproductive ability) with a $10 \%$ DNA methylation level, while mature tree zones demonstrated greater percentages that depended on their reproductive ability; the mature vegetative shoots showed $12.5 \%$ methylation. Floral differentiation implied a gentle decrease of genomic DNA methylation (12\%). Bud dormancy was accompanied with a $20 \%$ genomic DNA methylation, independently of the buds' ontogenic state. Hasbún $e t$ al. (2007) also studied the methylation increase in the epicormic apex during the reinvigoration process, which led to the loss of the reinvigoration effect until reaching a value near the observed one of the mature vegetative shoots (approximately after 3 growth seasons).

The changes in DNA methylation that occur in chestnut trees during their development and maturation were in agreement with published papers for other genera. Baurens et al. (2004) reported that the genomic DNA of microshoots with juvenile leaf morphology had a higher proportion of methylated cytosines than microshoots with mature phyllode-type in Acacia mangium Willd. According to Hasbún et al. (2005) the observed methylation of juvenile like stages in Castanea sativa was greater than the one observed at mature stages.

Furthermore, the SAM (shoot apical meristem) in higher plants, such as peach, represents the site at which organs are initiated and the growth pattern of the aerial plant body is established. Changes in the pattern of cellular differentiation and organ formation are strictly regulated by genes in the SAM and are activated during the transition from the juvenile phase to the vegetative or the reproductive-adult one (Bitonti et al., 2002). DNA methylation levels alter due to changes in both gene expression and chromatin structure. DNA methylation is also involved in gene regulation during developmental phases (Bitonti et al., 2002; Zhang et al., 2014).

Bitonti et al. (2002) studied the methylation differences between juvenile, juvenile-like (their morphology resembles the one of juvenile shoots) and adult shoots. Adult shoots revealed higher methylation levels with a wider distribution over the nucleus in comparison with the nuclei of juvenile and juvenile-like SAMs. The results indicate a hypermethylation of specific chromatin domains (such as highly CpG-rich sequences) in adult meristems than in juvenile ones. Hence, the level of methylation can have pronounced effects on development (Bitonti et al., 2002). Moreover, a peripheral pattern was prevalent in juvenile meristem nuclei, whereas a diffuse signal was predominant in adult meristem nuclei. These patterns coincide with the differences in the structural organization of chromatin in these types of meristems (juvenile and adult meristems).

Moreover, the flowering induction of Citrus, the most economically important fruit crop in the world, has been investigated for many years. Precocious trifoliate orange which has derived from trifoliate orange (Poncirus trifoliata L. Raf), has a short juvenile phase. Several citrus homolog 
genes such as LEAFY (LFY), APETELAI (AP1), TERMINAL FLOWER (TFL1), FLOWERING LOCUS $T$ (FT), APETELA3 (AP3), WUSCHEL (WUS), and FLOWERING LOCUS C (FLC) have been isolated and studied. Particularly, $L F Y$ is considered to be the central regulator of the flowering regulatory network (Zhang et al., 2014). The $L F Y$ protein is necessary for the vital transition from the vegetative to the reproductive developmental stage in flowering plants. If it is over-expressed it induces early flowering in both annual and perennial plants (Weigel and Nilsson, 1995; Blazquez et al., 1997).

5-Azacytidine (5-AzaC) is a pyrimidine nucleoside analog of cytidine that undergoes incorporation into DNA and blocks DNA methyltransferase; thus leading to hypomethylation and potentially beneficial re-expression of abnormally silenced genes. Additionally, it reduces the overall level of DNA methylation in chromatin. In annual plants, phenotypic changes induced by 5 -AzaC have been reported in Arabidopsis (Zhang et al., 2014). The results from applying 5 -AzaC to precocious trifoliate orange seeds showed that demethylation treatment directly or indirectly influences the expression of the genes CiFt, CiTFL1, CiLFY, CiAPI and CiFLC, during early flowering process. The abnormal plants have a decreased stature; smaller leaves, leaves with margins curled toward the upper leaf surface, as well as reduced apical dominance. Therefore, Zhang et al. (2014) supposed that the developmental abnormalities were correlated with altered patterns of gene expression. The only $\mathrm{CpG}$ island detected was located at the 5'-UTR tail of CiLFY, thus indicating that this gene may be regulated directly by DNA methylation (Zhang et al., 2014). Observations revealed that the overall DNA methylation status of CiLFY was $25.7 \%$ and $18.1 \%$ at juvenile and adult stages, respectively. The proportion of three different methylation contexts for juvenile stage was CpG 52.3\%; CpHpH 14.8\%; CpHpG 9.1\% and for adult stage was $\mathrm{CpG} 46.2 \%$; $\mathrm{CpHpH} 4 \%$; $\mathrm{CpHpG} 4 \%$, respectively. These results indicated the prominent methylation decrease during the transition from the juvenile stage to the adult stage in precocious trifoliate orange. The results also suggested that there may be an association of CiLFY demethylation, with the phase transition in precocious trifoliate orange (Zhang et al., 2014).

Finally, when investigating the changes in developmental stages in Malus domestica Borkh, Kumar et al. (2016) observed that the percentage of fully methylated fragments gradually decreased along with the dormancy break and fruit set under high chill conditions.

\section{Anthocyanins (flavonoids)}

In plants, flavonoids are synthesized through a balanced pathway yielding different subclasses of flavonoid compounds such as anthocyanins and carotenoids. In particular, anthocyanins are colored water-soluble pigments (in glycosylate forms) belonging to the phenolic group and they are responsible for the colors, red, purple, and blue in fruits and vegetables (Khoo et al., 2017). Each flavonoid compound serves different functions in plant development, reproduction, defense and protection against biotic/abiotic stresses (El-Sharkawy et al., 2015). Anthocyanin accumulation is based on: 1) its biosynthetic pathway with the numerous enzymes that catalyze it, 2) its transcription regulation that coordinates its induction during the fruit development or as a response to several environmental impacts (light, hormones etc.), and 3) its transportation from cytosol, where it is synthesized, to the vacuole, where it is stored (Cheng et al., 2014; El-Sharkawy et al., 2015). This localization is necessary for anthocyanins in order to function as pigments and to prevent them from being oxidized (Telias et al., 2011; El-Sharkawy et al., 2015). The basic structure of anthocyanins is composed of an anthocyanidin, a glycone and one or more sugar moieties linked to hydroxyl groups $3,5,7,39$, and 59 , with the $3^{\text {rd }}$ position on the C-ring being dominant (Cheng et al., 2014).

Anthocyanins are important secondary metabolites which serve to protect plants against pathogenic attack and UV radiation. They also provide flowers and fruits with pigmentation to attract pollinators and seed dispersers (Winkel-Shirley, 2001; Khoo et al., 2017). Methylation is known to affect both the stability and water solubility of anthocyanins; thus, it plays an important role in the accumulation of anthocyanins in plants. Anthocyanin methylation is catalyzed by anthocyanin Omethyltransferase (AOMT), and AOMT genes are responsible for the O-methylation of anthocyanins at either the 3' or the 5' position (Cheng et al., 2014).

The anthocyanins in apples do not only determine the peel's colour, but they are also a major part of their antioxidants (Tagliasacchi et al., 2007). With different types and/or numbers of sugar moieties attached to various positions, the structural diversity of anthocyanins increases significantly. Besides glycosylation, modifications such as methylation and acylation also contribute to the structural diversity of anthocyanins (Cheng et al., 2014). Consumption of apples in general has been associated with lower risks of cancer and cardiovascular diseases mostly due to their oxidative processes. Antioxidants are mainly localized in the apple peel (polyphenolics including anthocyanins), but their distribution patterns vary amongst the cultivars (Telias et al., 2011; Fang, 2015).

In apples, anthocyanins are predominantly represented by cyaniding-3-galactoside form, which is the major flavonoid induced during fruit maturation (Telias et al., 2011; El-Sharkawy et al., 2015). In 1993, Cocciolone and Cone reported that stripped patterns of anthocyanin accumulation in maize were a result of the differentially methylated 3' untranslated region of Pl-Bh, a MYB transcription factor which regulates anthocyanin accumulation. Since then at least three families, MYB, bHLH and WDR have been identified to be involved in the regulation of anthocyanin synthesis; however, the specific classes and genes involved vary depending on the species (Telias et al., 2011).

Transcript levels of the MYB1 allele correlate with anthocyanin accumulation and are higher in red fruit peel sectors (more exposed to light) and red peel cultivars than in green peel sectors of cultivars. Telias et al. (2011) showed that the variation in pigment accumulation between red and green stripes correlates with the anthocyanin levels and the steady state of mRNA levels in both anthocyanin 
biosynthetic genes and the transcription factor MYB10. In particular, red stripes were associated with lower methylation across the promoter of MYB10 in cv. 'Honeycrisp' and to a lesser degree in cv. 'Royal Gala'. In both cultivars, analysis showed that MYB10 promoter is highly methylated (above 60\%). Moreover, in the green stripes of 'Honeycrisp' the promoter had higher methylation levels than the red ones did (Telias et al., 2011). The transcript levels increased in dark-brown apples once exposed to light thus providing further evidence of its role as an anthocyanin regulator (Telias et al., 2011).

Bisulfite treatment and sequencing revealed that different cytosine contexts do not exhibit distinct methylation patterns, all cytosine contexts showed high methylation levels in highly methylated regions and vice versa. The $\mathrm{CpHpH}$ sites exhibited the highest percentage of methylation (20.2\%), followed by the $\mathrm{CpHpG}$ sites (16.9\%), while the CpG sites demonstrated the lowest percentage of methylation (1.6\%). Telias et al. (2011) associated DNA methylation in the promoter of MYB10 with reduced transcript accumulation. Their results also suggested that high levels of methylation in certain promoter regions of a key transcription factor in the flavonoid biosynthetic pathway in apple may play a regulatory role, without inhibiting gene activity.

Comparative analysis of the transcriptome of mutant $\mathrm{cv}$. 'Blondee' and its parent 'Kidd's D-8' (also known as 'Gala'), revealed that the loss of colour in 'Blondee' is a consequence of the collective repression of 34 genes (members of a WGCNA module 'Pink') which are highly associated with the anthocyanin content (El-Sharkawy et al., 2015). Genes MdMYB10 and MdGST, which are among the most suppressed in 'Blondee', were sequenced and their methylation status was analyzed. According to the analysis, the promoter regions MR3 and MR7 of MdMYB100 -the master regulator of anthocyanin in apple- were starkly differentially methylated, thus causing the color difference between 'Blondee' and 'Kidd's D-8'. Further analysis demonstrated that the methylated MR3 region reduced the binding of proteins MYB and MYC as well as the impact of the environmental stimulators. Telias et al. (2011) also observed that DNA methylation may affect MYB10 transcription through either by interfering with the RNApolymerase transcription complex or by preventing binding of additional factors required for transcription. Finally, methylation of MR3 and MR7 regions gradually increased during the fruit development, suggesting that the methylation of MdMYB10 is developmentally controlled (El-Sharkawy et al., 2015).

Peach (Prunus persica), a member of the Rosaceae family, is the third most important deciduous fruit trees worldwide. Peach is diploid $(2 n=2 x=16)$ with a small genome size of approximately $230 \mathrm{Mb}$ (Arús et al., 2012) and was first domesticated in the region of northwestern China between the Tarim basin and the north slopes of the Kunlun Shan Mountains. There are six types of anthocyanins in peach, and their structural diversity is attributed to glycosylation and/or methylation. Cheng et al. (2014) identified six anthocyanin-types in flowers of peach and its wild relatives. Peach and $P$. ferganensis demonstrated a similar anthocyanin composition in flowers, differing from both $P$. davidiana and $P$. kansueasis. Peach seems to be closely related to $P$. ferganensis and it is likely that peach is domesticated from $P$. ferganensis, a finding that is supported by the recent genome sequencing of peach (Arús et al., 2012). Within seven peach cultivars the anthocyanin composition did not vary, which is consistent with the narrow genetic base of peach (Cheng et al., 2014). Methylation and glycosylation contribute to the diversification of anthocyanins in peach. While four UGT genes are likely responsible for the glycosylation of anthocyanins, only one gene (PpAOMT2) is involved in the methylation of anthocyanins in peach. The spatiotemporal control of expression of these anthocyanin modification genes is considered to be responsible for the different patterns of anthocyanin accumulation in flowers, leaves, and fruit tissues (Cheng et al., 2014).

\section{Fertilization and Perpetuation (synergids and desiccated seeds)}

Tagliasacchi et al. (2007) studied the distribution patterns of DNA methylation between the two synergids in the embryo sac of Malus domestica Borkh, during two different stages before fertilization. Their research was based upon the fact that DNA methylation levels are specific for particular cell types but they may differ at times and within different genes. Synergids are complex cells of the female gametophyte. In angiosperms, the degeneration of one or occasionally both synergids near the time of fertilization, has been documented even though in some species one or both synergids can survive for a long time (Tagliasacchi et al., 2007).

During Stage I - before fertilization - mature synergids did not show structural differences nor did they demonstrate differences in their levels or distribution patterns of methylation (Tagliasacchi et al., 2007). At Stage II -near the time of fertilization- the micropylar synergid began to degenerate and the two synergids differed in their levels and distribution patterns of DNA methylation. The degenerating synergid started shrinking and its methylation levels were higher. According to Tagliasacchi et al. (2007), these data may indicate that the de novo DNA methylation of the degenerating synergid in apple, may induce the repression of some genes and the expression of other genes through the DNA demethylation process. The methylation of the degenerating synergid which may be necessary for the reproduction of apple fruits, takes place gradually as it has been observed during oocyte growth in mammals (Reik et al., 2001).

It is hypothesized that the increase in DNA methylation and its differentiated pattern in the degenerating synergid of Malus may be one of the factors responsible for the phenomenon of senescence of this cell (Tagliasacchi $e t$ al., 2007). DNA methylation seems to increase with the senescence in certain plant tissues which are determinant in specific phases of plant development; as it has been observed in the suspensor cells of Phaseolus coccineus (Andreucci et al., 1994; Pierotti et al., 1998) and in the tapetum of Malus domestica (Forino et al., 2003). The high level of DNA methylation in these cells may be able to enhance processes 
related to plant reproduction and to the first phases of embryo development, leading the cells to synthetize only those substances that is necessary for particular metabolic requirements (Tagliasacchi et al., 2007).

In 2013, Michalak et al. studied the methylation levels of Pyrus communis' desiccated seeds. The mechanisms of desiccation tolerance of orthodox (desiccation tolerant) seeds include the accumulation of late embryogenesis abundant proteins (LEA), dehydrins, non-reducing sugars, sucrose, selenium, activation of antioxidants, intracellular dedifferentiation, metabolic 'switching off and the operation of repair systems. It has been recently shown that epigenetic regulation of gene expression is involved in plants' response to environmental stresses (i.e. drought stress). It is apparent that plants modulate their physiology and development through epigenetic modifications of their DNA (Michalak et al., 2013).

Orthodox seeds are dried to specific moisture content before they are stored in gene banks that have been established to preserve the seeds under conditions that ensure genetic stability and longevity. Both seed moisture content and temperature, affect the course of epigenetic changes, including the global methylation of genomic DNA. Most of the stress-induced alterations in epigenetic modification of DNA are reset upon relief from the stress. However, in non-Mendelian inheritance, some of the modifications are stable and may be carried forward in the form of a 'stress memory' that is inherited across mitotic or even meiotic cell divisions. DNA methylation may be altered at whole-genome level as well as at the level of individual genes, in response to environmental stresses (Michalak et al., 2013).

According to Michalak et al. (2013), genome-wide changes in DNA methylation were induced in seeds after periods of severe desiccation. The desiccation of common pear seeds from 8.2 - 8.8\% to 4.8 - 5.3\% moisture content, resulted in a small decline of global DNA methylation while it did not affect the seed's germination or the emergence of seedlings. In highly desiccated seeds $(2.2-2.8 \%$ moisture content) an increase of the global DNA methylation levels was observed. Thus, it seems reasonable to conclude that seeds may attempt to survive extremely unfavorable environmental conditions by using hypermethylation of DNA, in order to reduce all vital cellular functions to a minimum. Moreover, a global increase in DNA methylation from 3.08 to $5.49 \%$ was observed in response to storage at $3{ }^{\circ} \mathrm{C}$ for 1 year (Michalak et al., 2013).

\section{Epigenetic diversity}

Prunus avium is an important forest tree with high quality wood production and a great ecological importance. It is partially asexual and presents discontinuous populations of scattered individuals which present a wealthy genetic diversity thus showing a high level of adaptability to their environments (Avramidou et al., 2015). The adaptive mechanism of dormancy in sweet cherry is believed to be associated with MADS-box genes (Rothkegel et al., 2017).

Many developmental processes have both genetic as well as epigenetic components, both of which determine the adaptability of an organism to altering environmental factors, including the anticipated climate change (Avramidou et al., 2015). Looking at the genetic and epigenetic diversity of populations of sweet cherry, Avramidou et al. (2015) spotted a lower diversity at epigenetic level than at the genetic one. In agreement with this finding was the work of Rico et al. (2014) who analyzed the genetic and epigenetic structures in natural populations of Quercus ilex. They observed higher (but not statistically significant) genetic diversity with $96.5 \%$ polymorphic loci at genetic level and $90 \%$ at the epigenetic level, while gene diversity was 0.37 and 0.31 respectively (Rico et al., 2014). In Prunus avium a non-significant correlation between geographic and epigenetic distances was spotted and it indicated the absence of strong effects due to region on epigenetic diversity. There was no significant correlation between genetic and epigenetic diversity of sweet cherry either, suggesting that different driving forces shaped these aspects of P. avium (Avramidou et al., 2015).

Rothkegel et al. (2017) analyzed the presence of matching siRNAs and DNA methylation in order to determine if PavMADS1 5' UTR intron presents epigenetic modification during the dormancy of sweet cherry. De novo methylation was observed in every cytosine context $(\mathrm{CpG}$, $\mathrm{CpHpG}$ and $\mathrm{CpHpH}$ ) and despite the increase in cytosine methylation at $888 \mathrm{CpHp}$, there were no matching siRNAs to this specific region. The increase of methylation could have been mediated by the hypermethylation of the promoter upstream and an increase of 24-nt siRNA matching this region (Rothkegel et al., 2017). According to recent studies in Arabidopsis, hypermethylated regions are considered to have induced the methylation of neighboring regions. During sweet cherry floral bud dormancy, the relative expression of genes PavDRM2.1 and PavDRM2.3 increase with chilling accumulation, suggesting that RdDM could be participating in dormancy regulation (Rothkegel $e t$ al., 2017). DNA methylation in promoter regions generally causes transcriptional gene silencing (Jones, 2012). Therefore, the promoter level results indicate that the RdDM pathway might be contributing to the silencing of PavMADS1 during floral bud dormancy since the CR is fulfilled. The results also indicate that PavMADS1 and PavMADS2 are candidates for negatively regulating PavFT expression and flowering and playing a key role in regulating the release of endodormancy in sweet cherry. The implication of siRNAs and epigenetic mechanisms in the regulation of PavMADS1 expression in sweet cherry floral buds is highlighted (Rothkegel et al., 2017).

\section{Summary}

Two forms of epigenetic control, DNA methylation and H4 acetylation, have been reported to play an important role in chestnut's bud burst and bud set (Santamaria et al., 2011). According to Hasbún et al. (2007) bud dormancy in Castanea sativa is accompanied with an increase in the global DNA methylation independently of aging. The methylation of cytosines $(\sim 20 \%)$ results in alterations in the transcriptional activity of DNA which leads to the inactivation of the meristems during the dormant period (Hasbún et al., 2007). Kumar et al. (2016) reported a 
progressive decrease of methylation along the chilling mediated dormancy release and subsequent fruit set in apple. In sweet cherry, during the floral bud dormancy, the relative expression of PavDRM2.1 and PavDRM2.3 increases with chilling accumulation, suggesting that RdDM could be participating in dormancy regulation, while DNA methylation in promoter regions has been reported to cause transcriptional gene silencing (Rothkegel et al., 2017).

Perennial plants undergo more than one change in their developmental stages within a year. It has been reported that DNA methylation either regulates or occurs during developmental changes in higher plants (Bitonti et al., 2002). The aging of chestnut has been associated with a gradual increase in DNA methylation (Hasbún et al., 2007). The vegetative shoots of chestnut demonstrate a higher percentage of methylation in comparison to juvenile shoots (Hasbún et al., 2007). During the active growth period, juvenile-like shoots were found to be more methylated than shoots from mature stages (Hasbún et al., 2005). Hasbún et al. (2007) reported an increase of methylation levels in chestnut during its development and the opposite effect during reinvigoration. Flowering, along other processes of dedifferentiation and cellular reprogramming, is associated with a decrease in methylation (Hasbún et al., 2005; Hasbún et al., 2007). In precocious trifoliate orange, the timing of the flowering process is regulated epigenetically, through DNA methylation, as the demethylation of CiLFY is considered to be the reason for gene expression during flowering initiation (Zhang et al., 2014). In Prunus persica, the adult shoot apical meristems (SAMs) demonstrated a strong zonation of chromatin condensation and DNA methylation that was not observed in juvenile or juvenilelike SAMs. This zonation in the adult SAMs is believed to be a result of the changes in gene expression due to DNA methylation (Bitonti et al., 2002).

Various flavonoid compounds are present in fruits; anthocyanins are considered to be of the most important ones. Anthocyanins have antioxidant and antiinflammatory functions, contributing to the protection of the fruit against biotic and abiotic stresses, traits that play an important role in the market acceptance of the fruits. In 'Blondee' apples (that lack the red colour), the 34 genes that have been associated with anthocyanins' expression were found to be repressed. The different anthocyanin levels of the red and the green stripes derive from the differential transcript accumulation of MYB10 (a master regulator of anthocyanin in apples), whose levels of methylation in its promoter region vary between these two states (Telias et al., 2011; El-Sharkary et al., 2015). Peaches are also rich in anthocyanins, having six types of anthocyanins whose structural differences are attributed to their different methylation or glycosylation state; as their spatiotemporal expression results in different patterns of anthocyanin accumulation in flowers, leaves and fruits (Cheng et al., 2014).

Another important developmental stage of fruit trees is the fertilization of flowers that will produce fruits. In Malus domestica high levels of methylation were observed in the degenerating synergid which appeared to be shrunk, a trait that may indicate one of the signals that lead to the activation of a dedifferentiated pathway thought to be responsible for the senescence of this cell (Tagliasacchi et al., 2007). The production of fruits plays a crucial role in the reproduction and the survival of the species. Inside most fruits, these seeds are present and have the ability to perpetuate the species. Moreover, these seeds have the ability to survive extremely unfavorable environmental conditions through DNA hypermethylation which causes the reduction of all vital cellular functions to a minimum (Michalak et al., 2013).

Finally, DNA methylation has been used to investigate the epigenetic diversity of sweet cherry. Although Avramidou et al. (2015) observed a high genetic differentiation; the epigenetic population differentiation was very weak. There was no significant correlation between genetic and epigenetic diversity.

\section{Conclusions}

To conclude, the role of methylation in perennial plants is evident in all of their processes as living organisms. Methylation plays an important role in bud dormancy, in the changes between developmental stages, in the expression of anthocyanins as well as fertilization and perpetuation. Bud dormancy is fundamental to the study of plant developmental processes and its regulation is of significant economic importance to fruit and horticultural industries. Temperate fruit trees -such as chestnut, sweet cherry and apple trees- undergo winter dormancy in order to survive from the low temperatures and short photoperiod. Dormancy has been characterized with a higher level of methylation in comparison to active growth periods. In apple trees, dormancy was accompanied with a decrease of the demethylation processes while in Prunus avium RdDM seems to regulate the dormancy. Genomic methylation could be considered as an epigenetic marker related to aging and reinvigoration and it has been demonstrated that it is essential for normal plant development. It also intervenes with the timing of bud activation and burst as well as the timing of the flowering process. Moreover, anthocyanins (an important flavonoid compound) are present at many tissues and especially fruits offering a wide range of functions (antioxidant, anti-inflammatory) and whose structural differences and expression are intertwined with methylation. Methylation plays an important role in the processes that are involved in plant reproduction and the senescence of particular cells. Furthermore, reports indicate that epigenetic phenomena such as DNA methylation are responsible for the reaction of orthodox seeds to sever desiccation. Finally, the driving forces that shape genetic diversity within a population do not have the same effect in the epigenetic level which presents little differentiation.

\section{References}

Andreucci AC, Ruffini-Castiglione M, Tagliasacchi AM (1994). RNA synthesis and immunogold localization of 5-methylcitosine rich regions in polytene chromosomes of Phaseolus coccineus L. embryo suspensor, at two stages of embryogenesis. Cytobios 78:99-113.

Arús P, Verde I, Sosinski B, Zhebentyayeva T, Abbott AG (2012). The peach genome. Tree Genetics and Genomes 8:1-17. 
10

Avramidou EV, Ganopoulos IV, Doulis AG, Tsaftaris AS, Aravanopoulos FA (2015). Beyond population genetics: natural epigenetic variation in wild cherry (Prunus avium). Tree Genetics \& Genomes 11(95):95.

Barghini E, Natali L, Cossu RM, Giordani T, Pindo M, ... Cavallini A (2014). The peculiar landscape of repetitive sequences in the olive (Olea europaea L.) genome. Genome Biology Evolution 6(4):776-791.

Baurens F, Nicolleau J, Legavre T, Verdeil J, Monteuuis O (2004). Genomic DNA methylation of juvenile and mature Acacia mangium micropropagated in vitro with reference to leaf morphology as a phase change marker. Tree Physiology 24(4):401-407.

Bitonti MB, Cozza R, Chiappetta A, Giannino D, Castiglione MR, ... Innocenti AM (2002). Distinct nuclear organization, DNA methylation pattern and cytokinin distribution mark juvenile, juvenilelike and adult vegetative apical meristems in peach (Prunus persica (L.) Batsch).Journal of Experimental Botany 53(371):1047-1054.

Blazquez MA, Soowal LN, Lee I, Weigel D (1997). LEAFY expression and flower initiation in Arabidopsis. Development 124(19):3835-3844.

Cheng J, Wei G, Zhou H, Gu C, Vimolmangkang S, ... Han Y (2014). Unraveling the mechanism underlying the glycosylation and methylation of anthocyanins in peach. Plant Physiology 166(2):1044 1058.

Chong S, Whitelaw E (2004). Epigentic germline inheritance. Current Opinion in Genetics \& Development 14(6):692-696.

Cocciolone SM, Cone KC (1993). Pl-Bh, an anthocyanin regulatory gene of maize that leads to variegated pigmentation. Genetics 135(2):575-588.

Cokus SJ, Feng S, Zhang X, Chen Z, Merriman B, ... Jacobsen SE (2008). Shotgun bisulfite sequencing of the Arabidopsis genome reveals DNA methylation patterning. Nature 452(7184):215-219.

Doerfler W, Bohm P (2006). DNA methylation: Basic mechanisms. Vol. 301. Springer Science \& Business Media.

El-Sharkawy I, Liang D, Xu K (2015). Transcriptome analysis of an apple (Malus $\times$ domestica) yellow fruit somatic mutation identifies a gene network module highly associated with anthocyanin and epigenetic regulation. Journal of Experimental Botany 66(22):7359-7376.

Fang J (2015). Classification of fruits based on anthocyanin types and relevance to their health benefits. Nutrition 31(11-12):1301-1306.

Flavell RB (1986). Repetitive DNA and chromosome evolution in plants. Philosophical Transactions of the Royal Society of London. B, Biological Sciences312(1154):227-242.

Forino LMC, Andreucci AC, Del-Tredici I, Felici C, Giraldi E, Tagliasacchi AM (2003). DNA methylation of tapetum cells during microsporogenesis in Malus domestica Borkh. Israel Journal of Plant Sciences 51(2):91-100.

Hasbún R, Valledor L, Berdasco M, Santamaría E, Cañal MJ, ... Sánchez M (2005). In vitro proliferation and genome DNA methylation in adult chestnuts. Acta Horticulturae 693:333-340.

Hasbún R, Valledor L, Santamaría E, Caña, MJ, Rodríguez R, Berdasco M (2007). Dynamics of DNA methylation in chestnut trees development. Acta Horticulturae 760(2):563-566.

Huh JH, Bauer MJ, Hsieh TF, Fischer RL (2008). Cellular programming of plant gene imprinting. Cell 132(5):735-744.

Jin B, Li Y, Robertson KD (2011). DNA methylation: superior or subordinate in the epigenetic hierarchy. Genes \& Cancer 2(6):607-617.

Jones PA (2012). Functions of DNA methylation: islands, start sites, gene bodies and beyond. Nature Reviews Genetics 13(7):484-492.

Kato M, Miura A, Bender J, Jacobsen SE, Kakutani T (2003). Role of CG and non-CG methylation in immobilization of transposons in Arabidopsis. Current Biology 13(5):421-426.

Katsiotis A, Hagidimitriou M, Douka A, Hatzopoulos P (1998). Genomic organization, sequence interrelationship, and physical localization using in situ hybridization of two tandemly repeated DNA sequences in the genus Olea. Genome41(4):527-534.

Kavarik A, Koukalova B, Bezdek M, Opatrny Z (1997). Hypermethylation of tobacco heterochromatic loci response to osmotic stress. Theoretical \&Applied Genetics 95(1-2):301-3069.

Khoo HE, Azlan A, Tang ST, Lin SM (2017). Anthocyanidins and anthocyanins: colored pigments as food, pharmaceutical ingredients, and the potential health benefits. Food \& Nutrition Research 61(1):121.

Kumar S, Cheng X, Klimasauskas S, Mi S, Posfai J, ... Wilson GG (1994). The DNA (cytosine-5) methyltransferases. Nucleic Acids Research 22(1):1-10.

Kumar G, Rattan UK, Singh AK (2016). Chilling-mediated DNA methylation changes during dormancy and its release reveal the importance of epigenetic regulation during winter dormancy in apple (Malusx domestica Borkh). PloS One 11(2):e0149934.

Lister R, Ecker JR (2009). Finding the fifth base: Genome-wide sequencing of cytosine methylation. Genome Research, Cold Spring Harbor Laboratory Press 19(6):959-966.

Liu I, Li Y, Tollefsbol TO (2008). Gene-environment interactions and epigenetic basis of human diseases. Current Issues in Molecular Biology 10(1-2):25-36.

Michalak M, Barciszewska MZ, Barciszewski J, Plitta BP, Chmielarz P (2013). Global changes in DNA methylation in seeds and seedlings of Pyrus communis after Seed Desiccation and Storage. PLOS ONE 8(8):e70693.

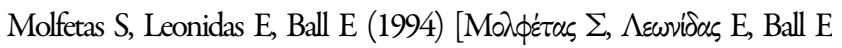
(1994)]. Biology: A trip to life. Genetics, 2. The Genetic Material and its Expression[In Greek]. pp 100-102.

Niederhuth CE, Schmitz RJ (2013). Covering your bases: Inheritance of DNA methylation in plant genomes. Molecular Plant 7(3):472-480.

Nikolaou C (2015). Computational Biology [In Greek]. Electronic Library pp 409-411.

Penterman J, Zilberman D, Huh JH, Ballinger T, Henikoff S, Fischer RL (2007). DNA methylation in the Arabidopsis genome. Proceedings of the National Academy of Sciences 104(16):6752-6757.

Pierotti L, Andreucci AC, Giraldi E, Tagiasacchi AM (1998). Polytene chromosomes of the embryo suspensor of Phaseolous coccineus L. during senescence. Cytobios 96:31-43.

Reik W, Dean W, Walter J (2001). Epigenetic reprogramming in mammalian development. Science 293(5532):1089-1093.

Reinders J, Wulff BBH, Mirouze M, Mari-Ordonez A, Dapp M, ... PaszkowskiJ (2015). Compromised stability of DNA methylation and transposon immobilization in mosaic Arabidopsis epigenomes. Genes \& Development 23(8):939-950. 
11

Rico L, Ogaya R, Barbeta A, Penuelas J (2014). Changes in DNA methylation fingerprint of Quercus ilex trees in response to experimental field drought simulating projected climate change. Plant Biology 16(2):419-427.

Rothkegel K, Sánchez E, Montes C, Greve M, Tapia S, ... Almeida AM (2017). DNA methylation and small interference RNAs participate in the regulation of MADS-box genes involved in dormancy in sweet cherry (Prunus avium L.). TreePhysiology 37(12):1739-1751.

Santamarna ME, Rodriguez R, Canal MJ, Toorop PE (2011). Transcriptome analysis of chestnut (Castanea sativa) tree buds suggests a putative role for epigenetic control of bud dormancy. Annals of Botany, Oxford University Press 108(3):485-498.

Singha S, Baugher T (2003). Concise encyclopedia of temperate tree fruit. New York: Food Products Press, pp 3-5.

Slavin JL, Lloyd B (2012). Health benefits of fruits and vegetables. American Society for Nutrition 3(4):506-516.

Smyth DR (1991). Dispersed repeats in plant genomes. Chromosoma 100(6):355-359.

Srinivasan PR, Borek E (1964). DNA modification mechanisms and gene activity during development. Science 187(4173):226-232.

Stergiou G, Katsiotis A, Hagidimitriou M, Loukas M (2002). Genomic and chromosomal organization of Ty1-copia-like sequences in Olea europaea and evolutionary relationships of Olea retroelements. Theoretical and Applied Genetics 104(6-7):926-933.
Tagiasacchi AM, Andreucci AC, Giraldi E, Felici C, Ruberti F, Forino LMC (2007). Structure, DNA content and DNA methylation of synergids during ovule development in Malus domestica Borkh. Caryologia 60(3):290-298.

Tahilliani M, Koh KP, Shen Y, Pastor, WA, Bandukwala H, ... Rao A (2009). Conversion of 5-Methylcytosine to 5-Hydroxymethylcytosine in Mammalian DNA by MLL Partner TET1. Science 324(5929):930935.

Telias A, Lin-Wang K, Stevenson DE, Cooney JM, Hellens RP, ... Bradeen JM (2011). Apple skin patterning is associated with differential expression of MYB1.BMCPlant Biology 11(93):1-14.

Tollefsbol T (2011). Handbook of Epigenetics: The New Molecular and Medical Genetics. London, UK, Burlington, USA, San Diego, USA.

Weigel D, Nilsson O (1995). A developmental switch sufficient for flower initiation in diverse plants. Nature 377(6549):495-500.

Winkel-Shirley B (2001). Flavonoid biosynthesis: a colorful model for genetics, biochemistry, cell biology, and biotechnology. Plant Physiology $126(2): 485-493$.

Xie M, Yu B (2015). siRNA-directed DNA methylation in plants. Current Genomic 16(1):23-31.

Zhang H, TangK, Wang B, Duan CG, LangZ,Zhu JK(2014). Protocol: a beginner's guide to the analysis of RNA-directed DNA methylation in plants. Plant Methods 10(1):18. 